\title{
Personalization of the Workplace through a Proximity Detection System Using User's Profiles
}

\author{
Carolina Zato, Alejandro Sánchez, Gabriel Villarrubia, Javier Bajo, \\ Sara Rodríguez, and Juan F. De Paz \\ Departamento Informática y Automática, Universidad de Salamanca, Salamanca, Spain \\ \{carol_zato, asanchezyu,gvg, jbajope,srg, fcofds\}@usal.es
}

\begin{abstract}
This article presents a proximity detection prototype that will be included in the future in an integral system primarily oriented to facilitate the labor integration of people with disabilities. The main goal of the prototype is to detect the proximity of a person to a computer using ZigBee technology and then, to personalize its workplace according to his user's profile. The system has been developed as an open MultiAgent System architecture using the agent's platform PANGEA, a Platform for Automatic coNstruction of orGanizations of intElligent Agents.
\end{abstract}

Keywords: proximity detection, Zigbee, RTLS, open MAS, agent platform, personalization, user's profiles, disabled people.

\section{Introduction}

Due to the advance of the technologies and communications, intelligent systems have become an integral part of many people's lives and the available products and services become more varied and capable, users expect to be able to personalize a product or service to meet their individual needs and will no longer accept "one size fits all". Personalization can range from simple cosmetic factors such as custom ring-tones to the complex tailoring of the presentation of a shopping web site to a user's personal interests and their previous purchasing behaviour [15]. It is expected to expand these innovative techniques to a wide range of fields. One of the segments of the population, which will benefit with the advance of personalized systems, will be people with disabilities [16], contributing to improve their quality of life [17]. Considering the near future, public and private companies will be provided with intelligent systems specifically designed to facilitate the interaction with the human users. These intelligent systems will be able to personalize the services offered to the users, depending on their concrete profiles. It is necessary to improve the services' supply, as well as the way to offer them [18]. Technologies such as Multiagent Systems and Ambient Intelligence based on mobile devices have been recently explored as a system of interaction with the 
dependent people [19]. These systems can provide support in the daily lives of dependent people [20].

This article presents a multi-agent based proximity detection prototype, specifically developed for a work environment, which can facilitate tasks such as activating and personalizing the work environment; these apparently simple tasks are in reality extremely complicated for some people with disabilities.

The rest of the paper is structured as follows: The next section introduces the detection proximity prototype, the technology used and how it works. Section 3 presents MAS in which the prototype is included. Section 4 explains the case study and finally, in section 5 some conclusions and future work are presented.

\section{Detection Proximity Prototype}

In this section we revise the proposed proximity detection prototype, focusing on the technology used and on the functioning of the prototype.

\subsection{Technology Used}

ZigBee sensors are used to deploy the detection prototype. ZigBee is a low cost, low power consumption, two-way wireless communication standard that was developed by the ZigBee Alliance [5]. It is based on the IEEE 802.15.4 protocol [2], and operates on the ISM (Industrial, Scientific and Medical) band at $868 / 915 \mathrm{MHz}$ and a $2.4 \mathrm{GHz}$ spectrum. Due to this frequency of operation among devices, it is possible to transfer materials used in residential or office buildings while only minimally affecting system performance [1]. Although this system can operate at the same frequency as Wi-Fi devices, the possibility that it will be affected by their presence is practically null, even in very noise environments (electromagnetic interference). ZigBee is designed to be embedded in consumer electronics, home and building automation, industrial controls, PC peripherals, medical sensor applications, toys and games, and is intended for home, building and industrial automation purposes, addressing the needs of monitoring, control and sensory network applications [5]. ZigBee allows star, tree or mesh topologies. Devices can be configured to act as network coordinator (control all devices), router/repeater (send/receive/resend data to/from coordinator or end devices), and end device (send/receive data to/from coordinator) [6]. One of the main advantages of this system is that, as opposed to GPS type systems, it is capable of functioning both inside and out with the same infrastructure, which can quickly and easily adapt to practically any applied environment.

Our prototype must allow performing efficient indoor locating in terms of precision because computers are very close one each other, for these reason the Real-Time Locating Systems (RTLS) Model was chosen. The infrastructure of a Real-Time Locating System contains a network of reference nodes called readers [12] and mobile nodes, known as tags [12][13]. Tags send a broadcast signal which includes a unique identifier associated to each tag. Then, readers obtain the identifier, as well as specific measurements of the signal. These measurements 
give information about the power of the received signal (e.g., RSSI), its quality (e.g., LQI, Link Quality Indicator), the Signal to Noise Ratio (SNR) or the Angle of Arrival (AoA) to the reader, amongst many others. These signals are gathered and processed in order to calculate the position of each tag.

RTLS can be categorized by the kind of its wireless sensor infrastructure and by the locating techniques used to calculate the position of the tags. This way, there is a range of several wireless technologies, such as RFID, Wi-Fi, UWB (Ultra Wide Band), Bluetooth and ZigBee, and also a wide range of locating techniques that can be used for determining the position of the tags [14].

\subsection{How the Prototype Works}

The proposed proximity detection system is based on the detection of presence by a localized sensor called the control point (where the ZigBeeReaderAgent is deployed), which has a permanent and known location. Once the Zigbee tag carried by the person has been detected and identified, its location is delimited within the proximity of the sensor that identified it. Consequently, the location is based on criteria of presence and proximity, according to the precision of the system and the number of control points displayed.

The parameter used to carry out the detection of proximity is the RSSI (Received Signal Strength Indication), a parameter that indicates the strength of the received signal. This force is normally indicated in $\mathrm{mW}$ or using logarithmic units $(\mathrm{dBm}) .0 \mathrm{dBm}$ is equivalent to $1 \mathrm{~mW}$. Positive values indicate a signal strength greater than $1 \mathrm{~mW}$, while negative values indicate a signal strength less than $1 \mathrm{~mW}$.

Under normal conditions, the distance between transmitter and receiver is inversely proportional to the RSSI value measured in the receiver; in other words, the greater the distance, the lower the signal strength received. This is the most commonly used parameter among RTLS.

RSSI levels provide an appropriate parameter for allowing our system to function properly. However, variations in both the signal transmission and the environment require us to define an efficient algorithm that will allow us to carry out our proposal. This algorithm is based on the use of a steps or measurement levels (5 levels were used), so that when the user enters the range or proximity indicated by a RSSI level of -50 , the levels are activated. While the values received are less than the given range, each measurement of the system activates a level. However, if the values received fall outside the range, the level is deactivated. When the maximum number of levels has been activated, the system interprets this to mean that the user is within the proximity distance of detection and wants to use the computer equipment. Consequently, the mechanisms are activated to remotely switch on both the computer and the profile specific to the user's disability.

The system is composed of 5 levels. The tags default to level 0 . When a user is detected close to a reader, the level is increased one unit. The perceptible zone in the range of proximity gives an approximate RSSI value of -50. If the user moves away from the proximity area, the RSSI value is less than -50 , resulting in a 
reduction in the level. When a greater level if reached, it is possible to conclude that the user has remained close to the marker, and the computer will be turned on.

On the other hand, reaching an initial level of 0 means that the user has moved a significant distance away from the workspace, and the computer is turned off.

The system uses a LAN infrastructure that uses the wake-on-LAN protocol for the remote switching on and off of equipment. Wake-on-LAN/WAN is a technology that allows a computer to be turned on remotely by a software call. It can be implemented in both local area networks (LAN) and wide area networks (WAN) [4]. It has many uses, including turning on a Web/FTP server, remotely accessing files stored on a machine, telecommuting, and in this case, turning on a computer even when the user's computer is turned off [7].

\section{System Architecture}

This proximity detection prototype is integrated within a open MAS that includes all the agents and information needed to create an integral system for helping disabled people in the workplace.

The open MAS has been created using PANGEA. There are many different platforms available for creating multiagent systems that facilitate the work with agents [8][9][10][11]; however our aim is to have a tool that allows users to create an increasingly open and dynamic multiagent system (MAS). PANGEA is a service oriented platform that allows to the implemented MAS to take the maximum advantage of the distribution of resources. To this end, all services are implemented as Web Services.

The own agents of the platform are implemented with Java, nevertheless the agents of the detection prototype are implemented in .NET and nesC.

Using PANGEA, the platform will automatically launch the following agents:

- OrganizationManager: the agent is responsible for the actual management of organizations and suborganizations. It is responsible for verifying the entry and exit of agents, and for assigning roles. To carry out these tasks, it works with the OrganizationAgent, which is a specialized version of this agent.

- InformationAgent: the agent is responsible for accessing the database containing all pertinent system information.

- ServiceAgent: the agent is responsible for recording and controlling the operation of services offered by the agents.

- NormAgent: the agent that ensures compliance with all the refined norms in the organization.

- CommunicationAgent: the agent is responsible for controlling communication among agents, and for recording the interaction between agents and organizations.

- Sniffer: manages the message history and filters information by controlling communication initiated by queries. 
These agents interact with the specific agents of the detection prototype:

- ZigbeeManagerAgent: it manages communication and events and is deployed in the server machine.

- UsersProfileAgent: it is responsible for managing user profiles and is also deployed in the server machine.

- ClientComputerAgent: these are user agents located in the client computer and are responsible for detecting the user's presence with ZigBee technology, and for sending the user's identification to the ZigbeeManager Agent. These agents are responsible for requesting the profile role adapted for the user to the ProfileManagerAgent.

- DatabaseAgent: the detection proximity system uses a database, which stores data related to the users, sensors, computer equipment and status, and user profiles. It can also communicate with the InformationAgent of PANGEA.

- ZigBeeCoordinatorAgent: it is an agent included in a ZigBee device responsible for coordinating the other ZigBee devices in the office. It is connected to the server by a serial port, and receives signals from each of the ZigBee tags in the system.

- ZigBeeReaderAgent: these agents are included in several ZigBee devices that are used to detect the presence of a user. Each ZigBeeReaderAgent is located in a piece of office equipment (computer).

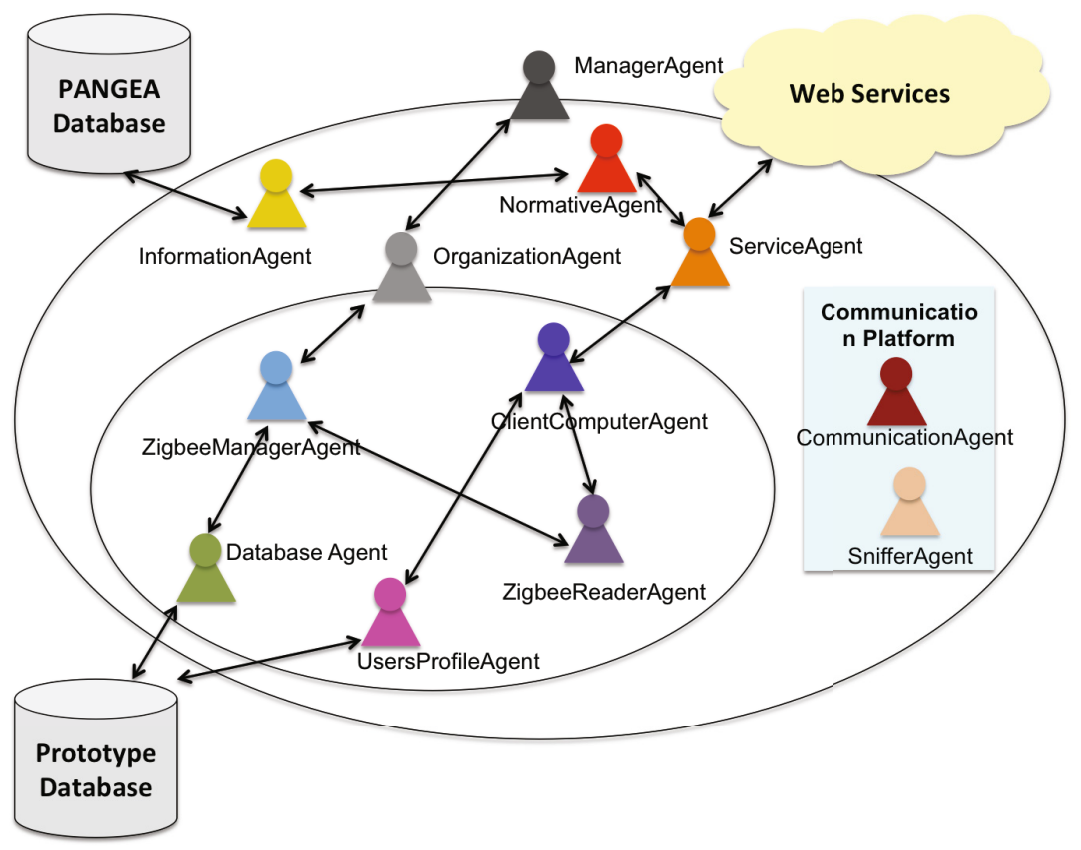

Fig. 1 System architecture 
Every user in the proposed system carries a Zigbee tag, which is detected by a ZigBeeReaderAgent located in each system terminal and continuously in communication with the ClientComputerAgent. Thus, when a user tag is sufficiently close to a specific terminal (within a range defined according to the strength of the signal), the ZigBeeReaderAgent can detect the user tag and immediately send a message to the ClientComputerAgent. Next, this agent communicates the tag identification to the UsersProfileAgent, which consults the database to create the $\mathrm{xml}$ file that is returned to the ClientComputerAgent. After, the ClientComputerAgent interacts with the ServiceAgent to invoke the Web Services needed to personalize the computer according to his profile.

\section{Case Study}

This paper presents a proximity detection system that is used by people with disabilities to facilitate their integration in the workplace. The main goal of the system is to detect the proximity of a person to a computer using ZigBee technology. This allows an individual to be identified, and for different actions to be performed on the computer, thus facilitating workplace integration: automatic switch on/off of the computer, identifying user profile, launching applications, and adapting the job to the specific needs of the user. Thanks to the Zigbee technology the prototype is notably superior to existing technologies using Bluetooth, infrareds or radiofrequencies, and is highly efficient with regards to detection and distance. Additionally, different types of situations in a work environment were taken into account, including nearby computers, shared computers, etc.

In our Case Study we have a distribution of computers and laptops in a real office environment, separated by a distance of 2 meters. The activation zone is approximately $90 \mathrm{~cm}$, a distance considered close enough to be able to initiate the activation process. It should be noted that there is a "Sensitive Area" in which it is unknown exactly which computer should be switched on; this is because two computers in close proximity may impede the system's efficiency from switching on the desired computer. Tests demonstrate that the optimal distance separating two computers should be at least $40 \mathrm{~cm}$.

Figure 2 shows two tools that the system provides in the main server, where the ZigbeeManagerAgent is running. The screen shown above allows tracking the flow of events and controlling which computers are on and who are the users, identifying their tags and consulting the UsersProfileManagerAgent. Moreover, this tool allows executing applications or programs remotely. Figure 3 shows the screen that appears in the user's computer when someone with a tag is close enough to it. The ClientComputerAgent running in this computer detects his presence and then, the computer is switched on with all the personal configurations. 


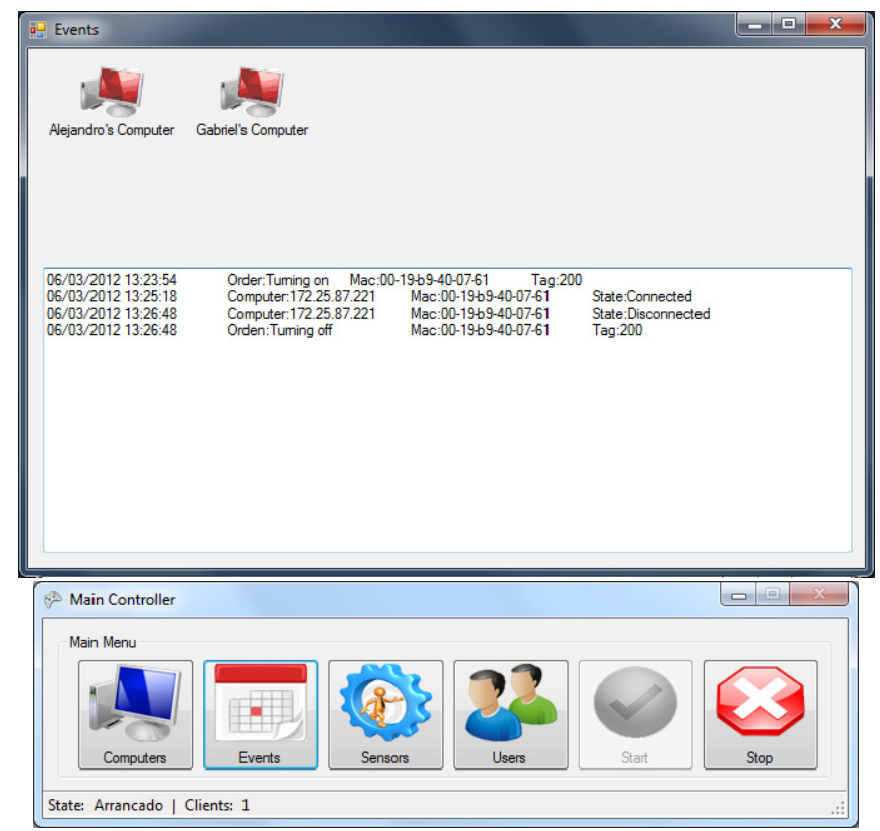

Fig. 2 Screenshot of the prototype in the main server

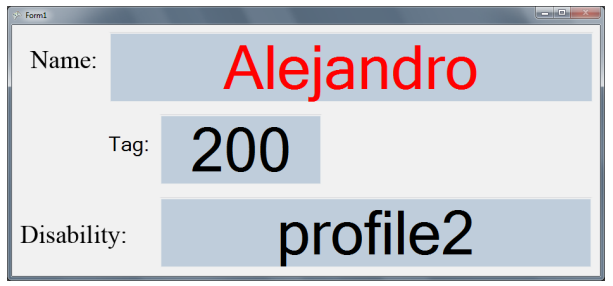

Fig. 3 Screenshot of the prototype in the main server

\section{Conclusions}

This prototype allows the detection and identification of a user making possible to detect any special needs, and for the computer to be automatically adapted for its use. This allows the system to define and manage the different profiles of people with disabilities, facilitating their job assimilation by automatically switching on or off the computer upon detecting the user's presence, or initiating a procedure that automatically adapts the computer to the personal needs of the user. This prototype is specifically oriented to facilitate the integration of people with disabilities into the workplace. 
The prototype is part of complete and global project in which different tools for helping disabled people will be included. Using the PANGEA, that models all the services as Web Services and promotes scalability, the addition in the future of all those services that conform the global project will be easier. Some of these future services include pointer services, predictive writing mechanisms, adaptation for alternative peripheral, virtual interprets in language of signs, identification of objects by means of RFID, etc.

Acknowledgements. This project has been supported by the Spanish CDTI. Proyecto de Cooperación Interempresas. IDI-20110343, IDI-20110344, IDI-20110345, and the MICINN TIN 2009-13839-C03-03 project. Project supported by FEDER funds.

\section{References}

1. Huang, Y., Pang, A.: A Comprehensive Study of Low-power Operation in IEEE 802.15.4. In: Proceeding of the 10th ACM Symposium on Modeling, Analysis and Simulation of Wireless and Mobile Systems, s.n., Chaina (2007)

2. Singh, C.K., et al.: Performance evaluation of an IEEE 802.15.4 Sensor Network with a Star Topology (2008)

3. Universidad Pontificia de Salamanca. [En línea] (2011), http: / / www . youtube. com/watch?v=9iYX-xney6E

4. Lieberman, P.: Wake on LAN Technology, White paper (2011), http: / /www. liebsoft.com/pdfs/Wake_On_LAN.pdf

5. ZigBee Standards Organization: ZigBee Specification Document 053474r13. ZigBee Alliance (2006)

6. Tapia, D.I., De Paz, Y., Bajo, J.: Ambient Intelligence Based Architecture for Automated Dynamic Environments. In: Borrajo, D., Juan, L.C., Corchado, M. (eds.) CAEPIA 2007, vol. 2, pp. 151-180 (2011)

7. Nedevschi, S., Chandrashekar, J., Liu, J., Nordman, B., Ratnasamy, S., Taft, N.: Skilled in the art of being idle: reducing energy waste in networked systems. In: Proceedings of the 6th USENIX Symposium on Networked Systems Design and Implementation, Boston, Massachusetts, April 22-24, pp. 381-394 (2009)

8. Agent Oriented Software Pty Ltd. JACK ${ }^{\mathrm{TM}}$ Intelligent Agents Teams Manual. s.1.: Agent Oriented Software Pty. Ltd. (2005)

9. Hübner, J.F.: J -Moise+ Programming organisational agents with Moise+ \& Jason. Technical Fora Group at EUMAS 2007 (2007)

10. Giret, A., Julián, V., Rebollo, M., Argente, E., Carrascosa, C., Botti, V.: An Open Architecture for Service-Oriented Virtual Organizations. In: Braubach, L., Briot, J.-P., Thangarajah, J. (eds.) ProMAS 2009. LNCS, vol. 5919, pp. 118-132. Springer, Heidelberg (2010)

11. Galland, S.: JANUS: Another Yet General-Purpose Multiagent Platform. Seventh AOSE Technical Forum, Paris (2010)

12. Liu, H., Darabi, H., Banerjee, P., Liu, J.: Survey of Wireless Indoor Positioning Techniques and Systems. IEEE Transactions on Systems, Man, and Cybernetics, Part C: Applications and Reviews 37(6), 1067-1080 (2007) 
13. Tapia, D.I., De Paz, J.F., Rodríguez, S., Bajo, J., Corchado, J.M.: Multi-Agent System for Security Control on Industrial Environments. International Transactions on System Science and Applications Journal 4(3), 222-226 (2008)

14. Tapia, D.I., Bajo, J., De Paz, J.F., Alonso, R.S., Rodríguez, S., Corchado, J.M.: Corchado Using Multi-Layer Perceptrons to Enhance the Performance of Indoor RTLS. In: Progress in Artificial Intelligence - EPIA 2011. Workshop: Ambient Intelligence Environmets (2011)

15. Pluke, M., Petersen, F., Brown, W.: Personalization and User Profile Management for Public Internet Access Points (PIAPs). CiteSeerX Scientific Literature Digital Library. Online Resource (July 2009), doi: 10.1.1.117.8111

16. Carretero, N., Bermejo, A.B.: Inteligencia Ambiental. CEDITEC: Centro de Difusión de Tecnologías, Universidad Politécnica de Madrid, España (2005)

17. Corchado, J.M., Bajo, J., Abraham, A.: GERAmI: Improving the delivery of health care. IEEE Intelligent Systems 23(2), 19-25 (2008)

18. Macarro, A., Bajo, J., Jiménez, A., de la Prieta, F., Corchado, J.M.: Learning System to Facilitate Integration through Ligthweigth Devices. In: Proceedings FUSION 2011, Chicago, US (2011) ISBN: 978-0-9824438-1-1

19. Anastasopoulos, M., Niebuhr, D., Bartelt, C., Koch, J., Rausch, A.: Towards a Reference Middleware Architecture for Ambient Intelligence Systems. In: ACM Conference on Object-Oriented Programming, Systems, Languages, and Applications (2005)

20. Ranganathan, V.K., Siemionow, V., Sahgal, V., Yue, G.H.: Effects of aging on hand function. Journal of the American Geriatrics Society 49, 1478-1484 (2001) 